\title{
DYNAMICS OF PERTURBATIONS AROUND INHOMOGENEOUS BACKGROUNDS IN THE HMF MODEL
}

\author{
J. Barréa $\underline{\text { A. Olivetti }}^{\mathrm{a}}{ }^{\dagger}$ and Y. Yamaguchi ${ }^{\mathrm{b}}$ \\ a'Laboratoire J.A. Dieudonné, Université de Nice Sophia-Antipolis \\ UMR CNRS 6621, Parc Valrose, F-06108 Nice Cedex 02, France \\ ${ }^{\mathrm{b}}$ Department of Applied Mathematics and Physics, Graduate School of Informatics \\ Kyoto University, Japan \\ ${ }^{\dagger}$ E-mail: Alain.OLIVETTI@unice.fr
}

The dynamical behavior and the relaxation to equilibrium of long range interacting systems of particles still offer several open problems. It is possible to sketch the general theory as follows: the short time behavior, on the order of the dynamical time scale, is described by the Vlasov, or a Vlasov-like equation. The system then settles in a quasi-stationary state (hereafter called QSS), which is a stationary solution of the Vlasov equation. On time scales diverging with the number of particles, the system evolves towards the thermodynamic equilibrium, following the analog of a Lenard-Balescu equation (for a recent review, see [1]). The open questions include for instance the selection of the QSS among the stationary states of the Vlasov equation; the possible selection of periodic solutions of the Vlasov equation instead of a QSS; the complete understanding of the timescales for relaxation to equilibrium, especially around inhomogeneous QSS and close to dynamical transitions. We may mention also the rigorous derivation of the Lenard-Balescu equation, which, in contrast to the Vlasov equation, does not have a mathematical foundation.

The stationary solutions of the Vlasov equation may be homogeneous in space, as is always the case in plasma physics, or inhomogeneous, as is the rule in self gravitating systems. In general, there is less understanding of the inhomogeneous cases, even at the level of linear perturbations of the Vlasov stationary state, because of the much greater technical difficulties. This linear understanding is an essential ingredient to derive a Lenard-Balescu like equation, and also to characterize possible undamped periodic solutions $[2]$.

The linearization around a homogeneous stationary state corresponds to the usual theory of Landau damping in plasma physics [3]. Technically, the equation are solved by a Fourier transform in space and a Laplace transform in time. The equations for different Fourier modes decouple, and the computation results in the complex dielectric function $\epsilon(k, \omega)$ for the $k$ th Fourier mode. Because $\epsilon(k, \omega)$ is defined in the upper half $\omega$-plane due to the convergence condition of the Laplace transform, one needs to analytically continue $\epsilon(k, \omega)$ in the lower half $\omega$-plane to obtain the Landau frequency and damping rate.

Linearization around a non homogeneous stationary state brings additional technical difficulties. First, one needs to use angle-action variables, to simplify the particle dynamics; but now the equations for different Fourier modes in the angle variables do not decouple any more; the "dielectric function" now becomes the determinant of an infinite matrix. Linearization around inhomogeneous stationary states has been studied in the context of self-gravitating systems, and, despite the technical difficulties, the above procedure has been carried out entirely to study the instability of some galactic models.

However, studying the analog of Landau damping requires the analytic continuation part, and this is, in the words of Weinberg [4], a "daunting task". This difficulty was by-passed in [4] by fitting $\epsilon(\omega)$ in the upper half $\omega$-plane with rational functions. This is the only article we are aware of where there is a quantitative study of gravitational Landau damping, with an explicit derivation of damping rates and frequencies. This may seem surprising, but the explanation probably lies in the dissuasive complexity of such computations.

In this presentation, we will be interested in the linearization around inhomogeneous stationary states of a Vlasov equation. To reduce as much as possible the technical difficulties, we will use the 1D HMF model, a much simpler setting than a 3D self-gravitating system. This will enable us to: 
- study a situation where the one-particle dynamics presents a separatrix

- explicitely perform the analytical continuation of the "dielectric function" $\epsilon$, and unveil the structure of its singularities; in particular, we show that it presents an infinite number of branch cuts (see figure 1)

- predict damping rate and oscillation frequency for a wide class of initial stationary distributions

- confront the results with direct N-body simulations.

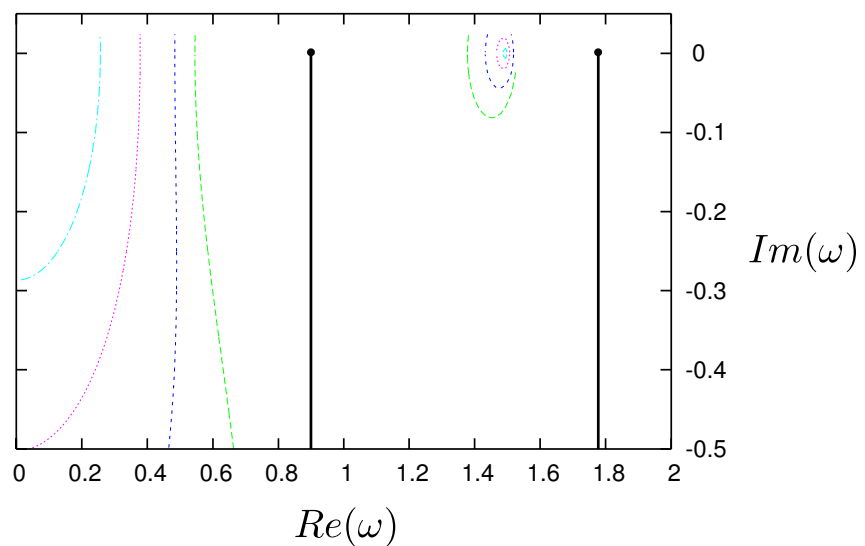

(a)

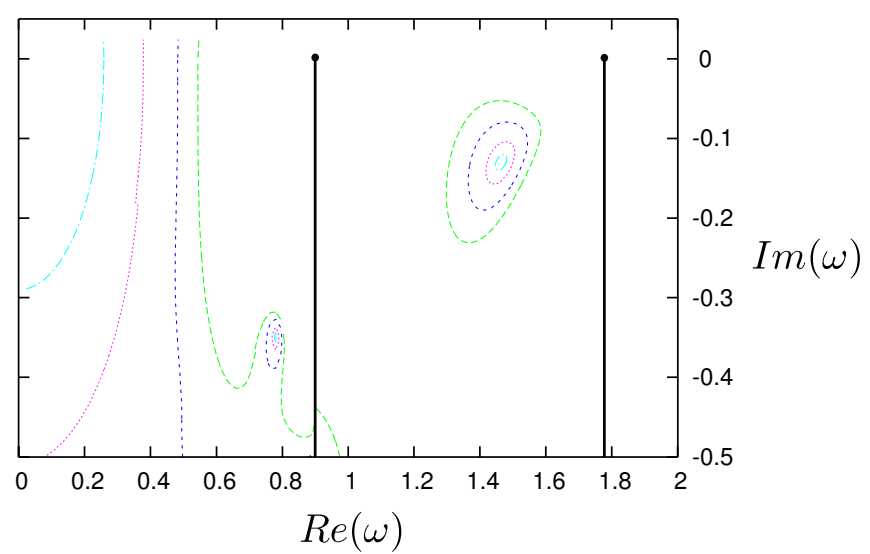

(b)

Figure 1: Level curve of $|\epsilon(\omega)|$ in $\omega$-complex plane for two inhomogeneous distributions. Magnetization is set to 0.8. Branch cuts are represented by vertical black lines. (a) Waterbag case; (b) Gaussian case.

\section{References}

[1] A. Campa, T. Dauxois and S. Ruffo, "Statistical mechanics and dynamics of solvable models with long-range interactions," Physics Reports, v. 480, 2009.

[2] J. Barré and YY. Yamaguchi, "Small traveling clusters in attractive and repulsive Hamiltonian meanfield models," Physical Review E, v. 79, 2009.

[3] L. Landau, "On the vibrations of the electronic plasma," Journal of Physics USSR, v. 10, 1946.

[4] MD. Weinberg, "Weakly damped modes in star clusters and galaxies," Astrophysical Journal, v. 421, 1994.

[5] M. Antoni, S. Ruffo, "Clustering and relaxation in Hamiltonian long-range dynamics," Physical Review $E$, v. $52,1995$. 